\title{
Numerical experiment about the arrangement determination of multiple bluff cylinders which obtain the most suitable new type stir
}

\author{
Yoshifumi Yokoi ${ }^{1,2, a}$ \\ ${ }^{1}$ National Defense Academy, Department of Mechanical Engineering, 1-10-20 Hashirimizu, Yokosuka 239-8686, Japan \\ ${ }^{2}$ Techniche Universität Berlin, Müller-Breslau-Str. 8, 10623 Berlin, Germany
}

\begin{abstract}
When developing the stirring rod for mixing two liquid with which the densities into which gas melted differ in little stirring operation, verification of the stirring effect was performed in the numerical simulation using a vortex method. Since it is calculation for specification examination of the preceding paragraph which creates a producing model, it becomes important to obtain a calculation result early and to feed back to the design. Two dimension calculation of the double file circular cylinder group which carried out staggered arrangement of the nine circular cylinders was performed. The aspects of the flow which strayed off from the design point or the design point were shown. The mixed degree was judged from the degree of the complexity of the flow pattern. In a design point, the complicated flow by a vortex is formed with oscillating 1 period, and interference stirring occurs. Adaptation ranges other than a design point are that the ranges of the value of $P / L$ are $0.8-1.2$.
\end{abstract}

\section{Introduction}

In the restaurant in Japan, various alcoholic beverages are offered like the countries of Europe. Beer, sake, white distilled liquor, wine, whiskey, etc. are prepared there. Consumption of plum wine with soda is increasing in such an alcoholic beverage. This is because plum wine with soda passes along a throat smoothly. In women or the younger age group, it is reported that the amount of consumption of plum wine with soda is increasing [1]. In response to this matter, alcoholic beverage brewing industry companies vied in production increase-inquantity sale of a "soda cocktail" drink mutually, and planned it. In order to attain differentiation on sale, the improvement idea of packaging, addition of a sales promotion article, etc. were planned. Development of a new type "muddler" was desired as one of the plans of differentiation on sale. Here, although the "muddler" is "cocktail stir" in use, it is the popular name currently called in usually in the food-and-drink industry of Japan. The popular name was used in this study. Generally, the "muddler" of the stirring use of the cocktail drink is a long and slender bar configuration. The method for use agitates by making it circle horizontally in the liquid in a glass. Since the liquid in a glass also rotates synchronizing with a muddler, in the shape of an ordinary muddler, stirring efficiency is low. Therefore, in order to obtain enough stirring effect, it is necessary to continue rotating a muddler over a long time. In a "soda cocktail", if it continues rotating a muddler over a long time, the

\footnotetext{
${ }^{\mathrm{a}}$ Corresponding author: yokoi@nda.ac.jp
}

demerit in which carbon dioxide falls off from the liquid by the centrifugal force produced in a motion of the muddler will occur. The good method of raising the efficiency of stirring should just make a muddler reciprocating movement within the glass horizontally. However, the method is not efficiently mixed over the overall fluid in the glass.

The author is observing that mix as the symmetrical vortex street discharged from two circular cylinders which are oscillating in the direction of a flow flows and goes down through study of a lock-in phenomenon until now, and rearrangement occurs as a vortex of another kind [2, 3]. By using the lock-in phenomenon and phenomenon of vortical rearrangement, the author conjectured what can promote liquid stirring. Then, the author performed creation development of the muddler (type shaken longitudinal) of the new shape based on interference stirring by vortices. And the muddler was used as the product and sent out to the market [1].

This paper reports the details which are the numerical simulations performed in the early stage of the creation development.

\section{Numerical calculations}

\subsection{Test design of the muddler for numerical simulations}

Mixture of ume liquor and carbonated water was assumed with the cylinder glass with a capacity of $460 \mathrm{ml}$ (the 
diameter of $70 \mathrm{~mm}$, the depth of $120 \mathrm{~mm}$ ) containing ice. In order to make it mix moderately in a little operation, a motion of the muddler makes it reciprocating movement in the perpendicular direction of the glass. In order to enlarge the contact surface product when moving in one operation in the inside of a liquid, the tip section of a stick made shape of a muddler the shape rolled spirally. It was considered as the shape of a coil. The shape is shown in figure 1. The circular cylinder which became this coil shape plans making a vortex, discharging and agitating surrounding fluid by the mutual interference of the discharged vortices. The muddler is inconvenient with the metal which rusts on a health concept target in order to touch a drink. It becomes a cost overrun although what is necessary is just to use stainless steel for the material. The cast made of resin which can be mass-produced at a low price also from the field of manufacture expense was assumed. In consideration of intensity and productivity, the diameter $d$ of a material round bar was determined as $4 \mathrm{~mm}$.

As for a vortex shedding occurring independently mutually, it is known for parallel two circular cylinder that the ratio $(G / d=(L-d) / d)$ of the circular cylinder interval $G$ and the circular cylinder diameter $d$ is 1.0 or more. In tandem two circular cylinders, it is known that the separating shear layer discharged from the upstream side circular cylinder at the time of about $P / d$ (ratio of circular cylinder center interval $P$ and circular cylinder

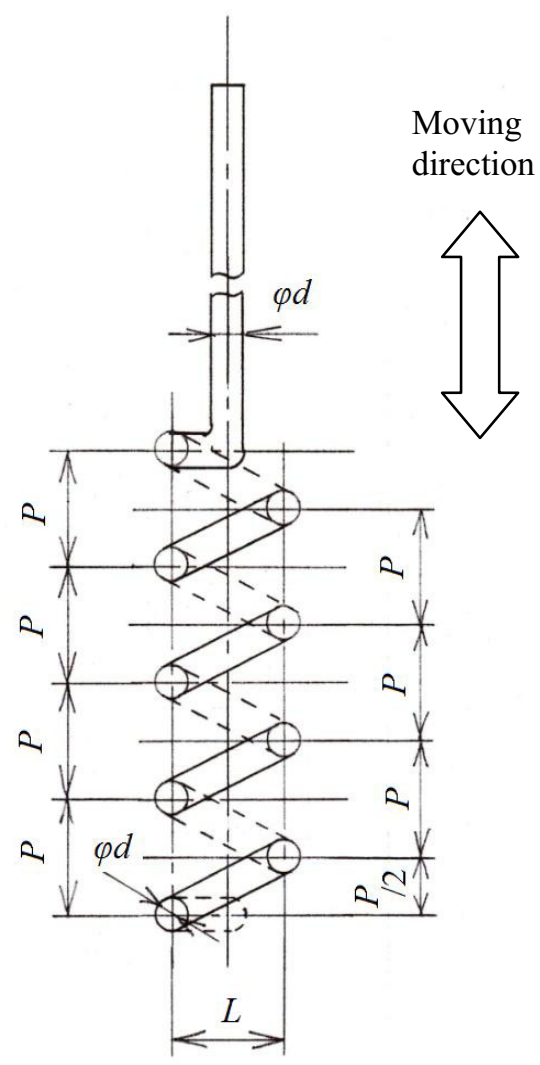

Figure 1. Muddler tip section shape

diameter $d)=2.5$ will carry out a reattachment to the downstream side circular cylinder [4]. In consideration of this thing, the design of the coil centre distance $L$ and the design of a coil pitch $P$ were performed. The value of $P / d$ was set to 3.5 in order to secure the space which has been rolled before the circular cylinder by the side of downstream by the separating shear layer discharged from the circular cylinder by the side of the upstream. The value of space ratio $P / L$ which is the ratio of a coil pitch $P$ and the coil centre distance $L$ was decided to be 1.0 (design point). As a result, it was decided that it would be $P=L=14 \mathrm{~mm}$. The length of the coil section was made into the 4 times roll $(4 P=56 \mathrm{~mm})$ in consideration of the appearance as the muddler. The conditions of oscillation were decided as follows. Half oscillating amplitude $a$ was set to $30 \mathrm{~mm}$ so that oscillating amplitude might become equivalent to the length of the coil section. Frequency $f$ of oscillation was set to $1 \mathrm{~Hz}$ in consideration of the cardiac beats rate of the human body at the time of drinking.

\subsection{The calculation method and apparatus}

The numerical simulation was performed by commercial fluid analysis tools and the commercial note type computer. The fluid analysis tools of the marketing used "Uzu Cruise 2D Ver.1.1.3 Rev.H" by College Master Hands, Inc. The numerical computation technique of the used fluid analysis tools is a vortex method which introduced the surface of wall vortex element method which is a type the vortex method application level 3 which used together the boundary element method and the vortex method developed. The details as the calculation technique of the vortex method are written to reference [5] and reference [6]. The computer used the commercial note type personal computer (NEC LaVie LL850/L).

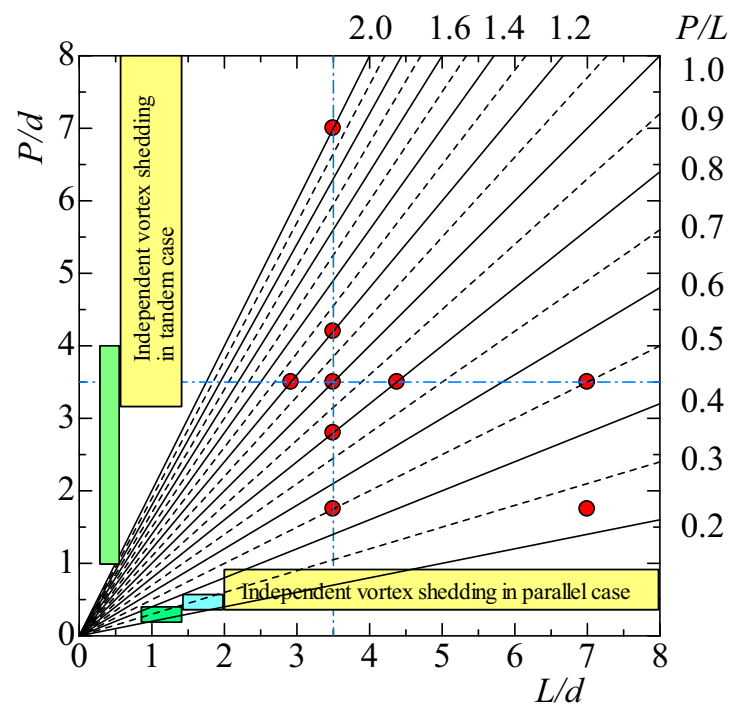

Figure 2. The diagram of the calculation points 


\subsection{Calculation conditions}

This calculation is calculation for specification examination of the preceding paragraph which creates a producing model. So, it is important to obtain the calculation result early and to feed back to a design. In this study, two dimensional calculation of the circular cylinder group which treated the section including the main axis of the coil, then carried out staggered arrangement of the nine circular cylinders was performed. The mixed degree was judged from the degree of the complexity of the flow pattern. Two kinds of liquids with which density differs from specific gravity in fact are mixed. However, in order to verify the process of mixture by the vortex, it was considered as single fluid and the physical-properties value of water was used for calculation here. Here, the spatial restriction supposing a glass, ice, etc. is not provided. The state of early fluid is stationary. Half oscillating amplitude was set to $a=30$ $\mathrm{mm}$, and the oscillation frequency was set to $f=1 \mathrm{~Hz}$. The Reynolds number ( $R e=u_{\max } \cdot d / v$ ) based on the maximum movement speed of body $\left(u_{\max }=2 \pi a f\right)$ was about 660. If the Strouhal number was calculated using the body oscillation frequency and amplitude, the value of the Strouhal number was about 0.02 .

In such the state, in order to investigate the applicability of space ratio $(P / L)$, the value of space ratio $(P / L)$ was varied and the simulation was performed. The value of space ratio $(P / L)$ was changed with $0.25,0.5,0.8$, $1.0,1.2$, and 2.0. The total number of times of repetition calculation is 1000 times, and this corresponds by 2.5 times the oscillating period. Figure 2 shows a design point and each calculating point in each space ratio with the aspect of the flow of parallel two circular cylinders, and the aspect of the flow of tandem two circular cylinders. An abscissa is the distance ratio in the case of parallel two circular cylinders, and an ordinate is the distance ratio in the case of tandem two circular cylinders. The straight lines in the figure mean the space ratio $(P / L)$.

\section{Result and discussion}

\subsection{Aspect of the flow in a design point}

The aspect of the flow for every time progress in a design point $(P / L=1.0, L / d=3.5, P / d=3.5)$ is shown in figure 3. Here, "Origin" is in the state of the calculation start. "T.D.C." and "B.D.C." mean the top dead center of oscillation, and the bottom dead center of oscillation, respectively. The "cycle" expresses one period and the numeric character in a parenthesis shows the number of calculation steps. Since preservation of calculated data was performed every ten steps, the flow pattern of each period, the top dead center, and the bottom dead center used the thing of the step near it. It is imagined that mixture of flow is promoted at the time of the turn to the bottom dead center from the top dead center and the turn to the top dead center from the bottom dead center. When it sees through oscillating one period, it turns out that the complicated flow by vortices was formed and interference stirring has occurred. Furthermore, the flow increased complexity by continuing oscillation. After

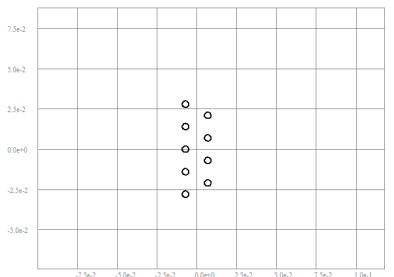

(a) Origin (0)

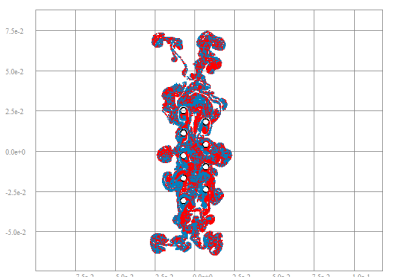

(e) 1 cycle $(360)$

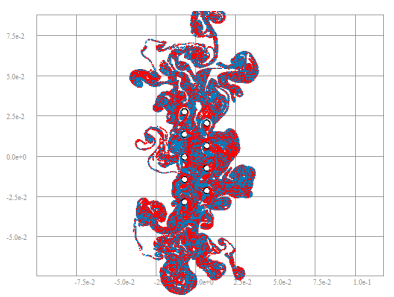

(i) 2 cycle (750)

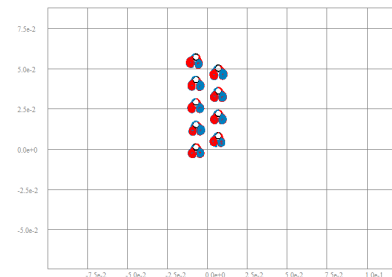

(b) T.D.C. (80)

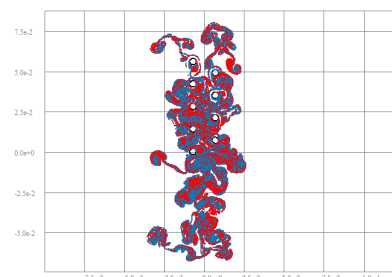

(f) T.D.C. (460)

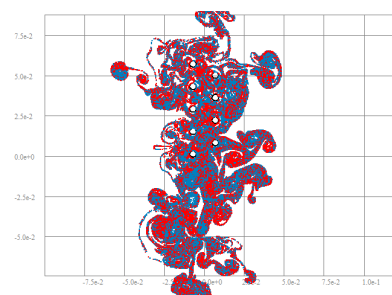

(j) B.D.C. $(850)$

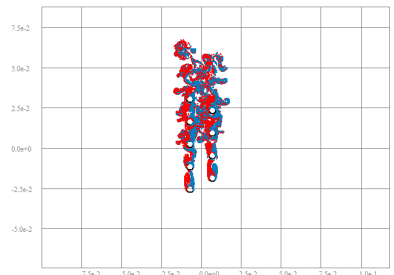

(c) 0.5 cycle (170)

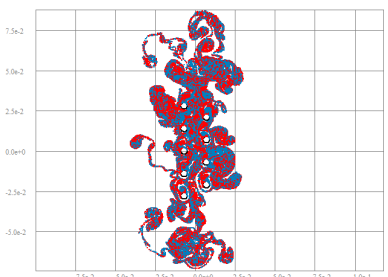

(g) 1.5 cycle $(560)$

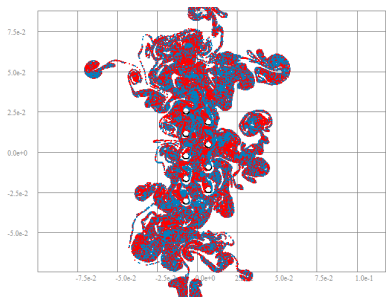

(k) 2.5 cycle $(950)$

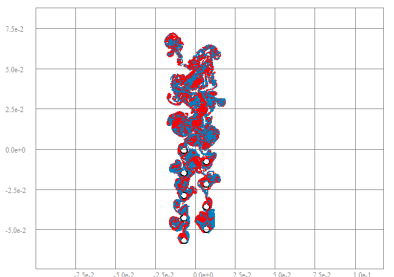

(d) B.D.C. (270)

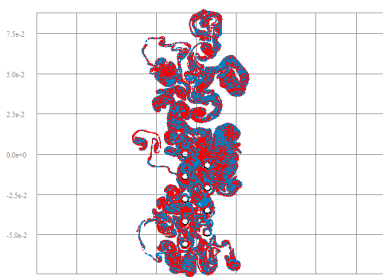

(h) B.D.C. (650)

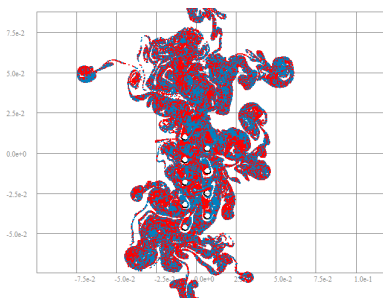

(1) Final calculation (1000)

Figure 3. The time history of the flow pattern in a design point $(P / L=1.0, L / d=3.5, P / d=3.5)$ 


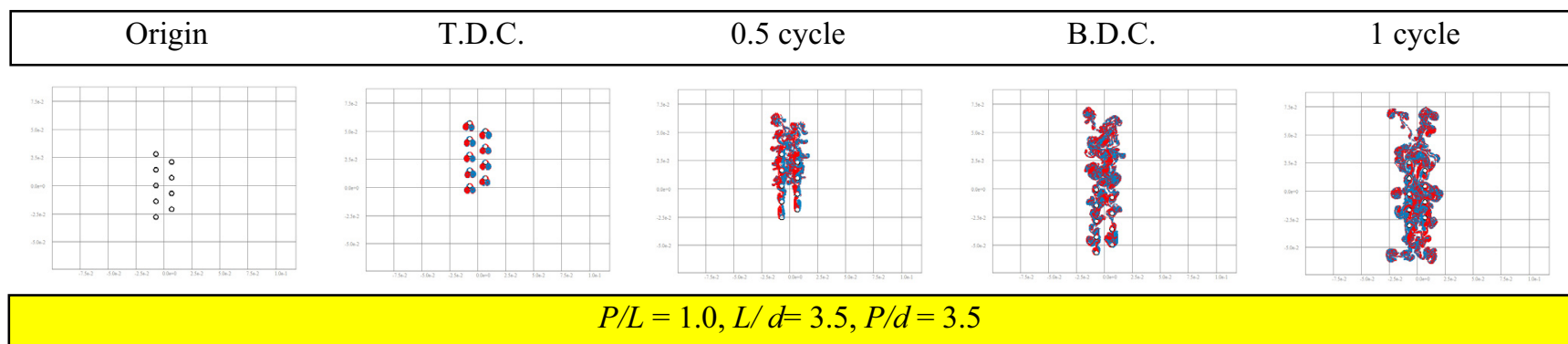

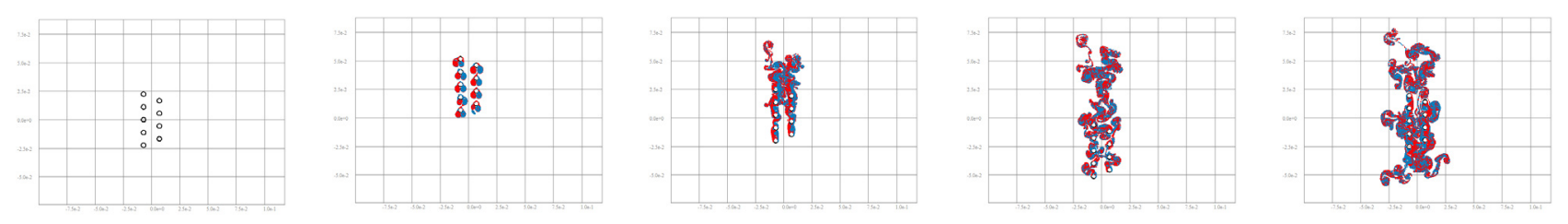

\section{$P / L=0.8, L / d=3.5, P / d=2.8$}
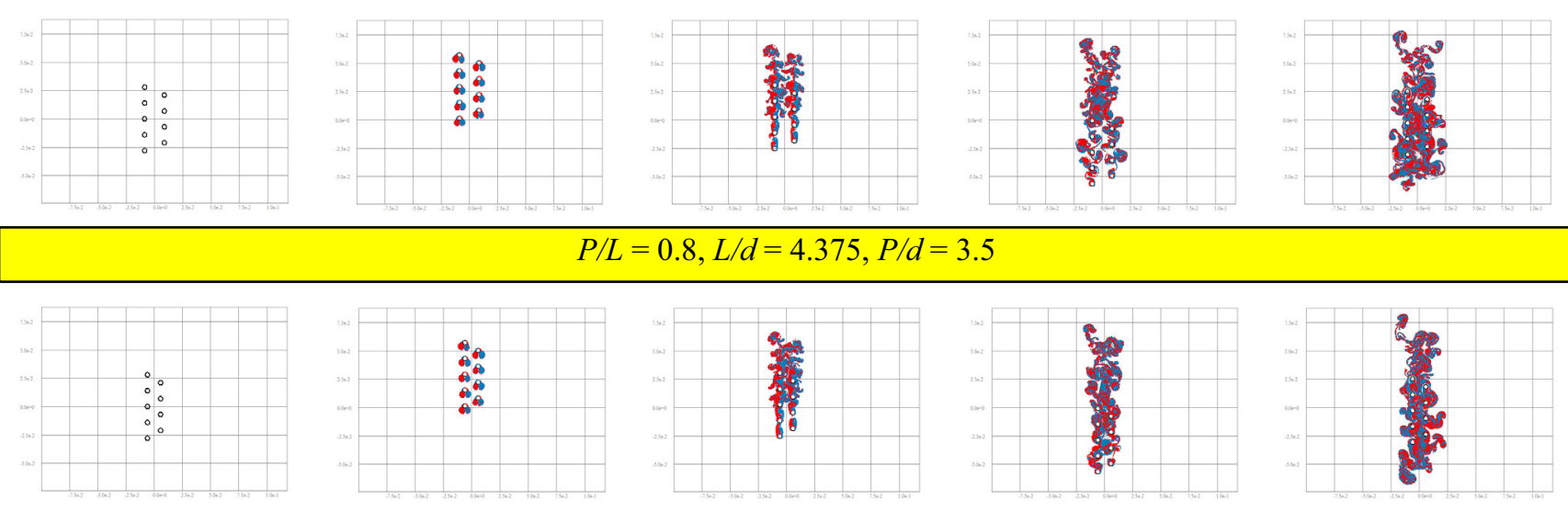

$$
P / L=1.2, L / d=2.917, P / d=3.5
$$

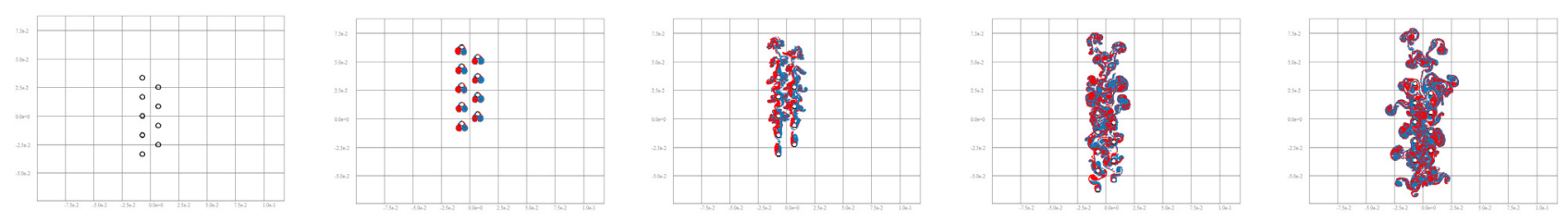

$$
P / L=1.2, L / d=3.5, P / d=4.2
$$

Figure 4. The time history of the flow pattern when straying off $\pm 20 \%$ from a design point $(P / L=0.8$ and 1.2$)$

passing over oscillating two periods, mixture by largescale vortices can be seen. This thing suggests that "good stirring" is obtained by little movement.

\subsection{Examination of the space ratio suitable for stirring}

The aspects of the flow of space ratios other than a design point are shown in figure 4 and figure 5. The aspect of the flow of the design point is also displayed on both figures for comparison. In the design point, the complicated developed flow is formed oscillating one period. Therefore, the flow patterns of the "initial state", the "top dead center", the "half cycle", the "bottom dead center", and the "one period" are shown here, respectively. The conditions currently colored by yellow all over the figure show the case where the vortices which became independent of each circular cylinder are discharged. Figure 4 shows the flow patterns of the conditions used as $P / L=0.8$ and $P / L=1.2$ which strayed off from the space ratio in a design point $\pm 20 \%$. On every condition, the complicated flow by a vortex was formed with oscillating one period, and interference stirring has occurred, and it can grasp so that it may scarcely change to the aspect of the flow in a design point. On the other hand, figure 5 shows the flow pattern in the case of the space ratio which strayed off from the design point further. When a result is seen, it turns out that enough on any conditions stirring is not obtained. These matters show that the ranges of $P / L$ of space ratio suitable for stirring are 0.8 1.2. Here, when it is on the line of the same space ratio, the one where the value of $L / d$ or $P / d$ is smaller is considered to be a desirable thing on the size of the product. 


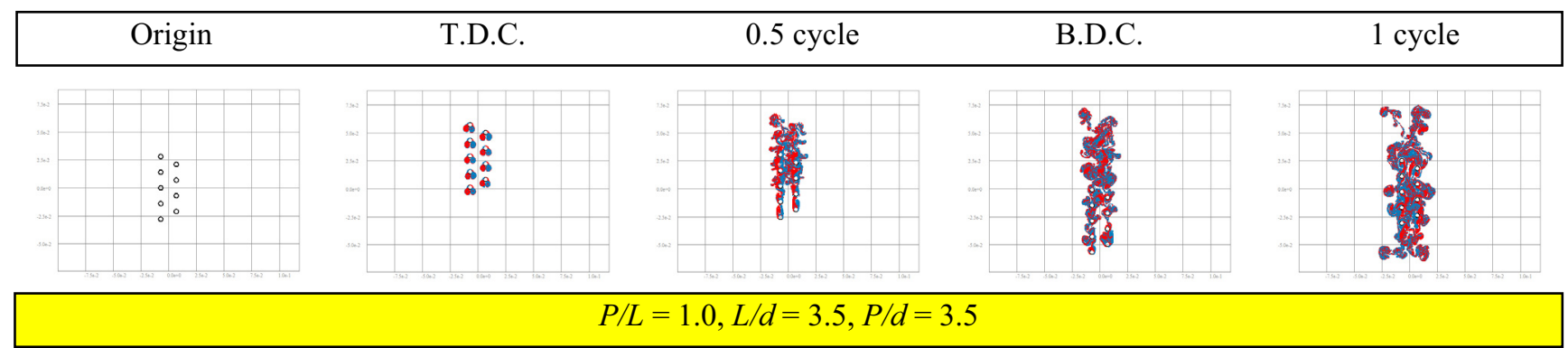
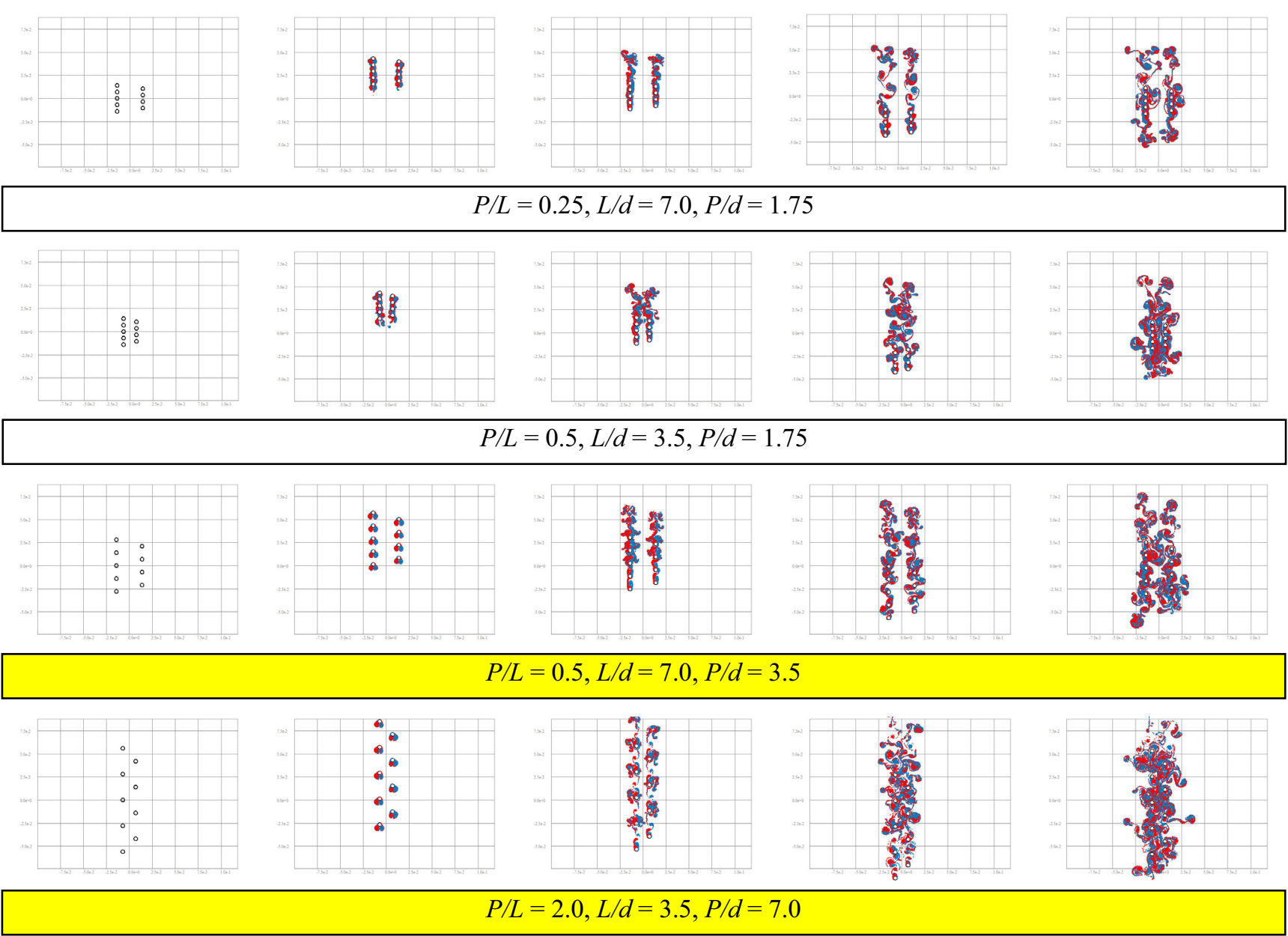

Figure 5. The time history of the flow pattern when straying off further from a design point $(P / L=0.25,0.5$ and 2.0$)$

\section{Conclusions}

In order to develop the stirring rod for mixing two liquid with which densities called sodium carbonate and ume liquor differ in little stirring operation, verification of the stirring effect was performed in the numerical simulation using the vortex method. The following conclusions were obtained.

(1) In a design point, a complicated flow is made from oscillation of the one period by vortices. And interference stirring occurs.

(2) Adaptation ranges other than the design point are that the ranges of the value of $P / L$ are $0.8-1.2$.

(3) The calculation using the vortex method is the simple technique whose product development is possible while making it feed back to the design.

\section{Acknowledgement}

An author expresses gratitude to Sapporo Breweries, Ltd., Daiko Advertising, Inc., and Avalanche, Inc. The opportunity to which an author carries out research from the staff of those companies was obtained. And they sent out the product borne by this research to the market. An author expresses deep gratitude to the staff members anew. 


\section{References}

1. T. Monna, Y. Yokoi, Proc. 50th all Japan packing technical research convention, 265-268 (2012)

2. Y. Yokoi, K. Hirao, Trans. Jpn Soc. Mech. Eng. 74748, 2466-2475 (2008)

3. Y. Yokoi, K. Hirao, Trans. Jpn Soc. Mech. Eng. 77775, 590-601 (2011)

4. JSME ed., JSME Standard S012, B78(1998)

5. K. Kamemoto, Computational Fluid Dynamics, 2-1, 20-29 (1993)

6. K. Kamemoto, Computational Fluid Dynamics, 2-2, 28-39 (1994)

\section{Appendix}

The data of "the new type muddler" which the author developed are attached. Figure 6 shows the blueprint and its finished goods. The material of parts is plastic resin. This article is the goods developed for advertisement sales promotion of ume liquor. Figure 7 shows the muddler attached to the package of ume liquor. The sale was performed by such packaging. Figure 8 shows the covering letter attached to the muddler. Since the campaign was performed in Japan, it is written in Japanese. The muddler is shown in the beer jug which a model woman (right-hand side) has in a hand. The author (left-hand side) is also introduced as a designer. Since "the cheers" was called "Kanpai" in Japan, this muddler was named the "Kanpai-muddler." Figure 9 is the back of the covering letter, and the method for use of the muddler

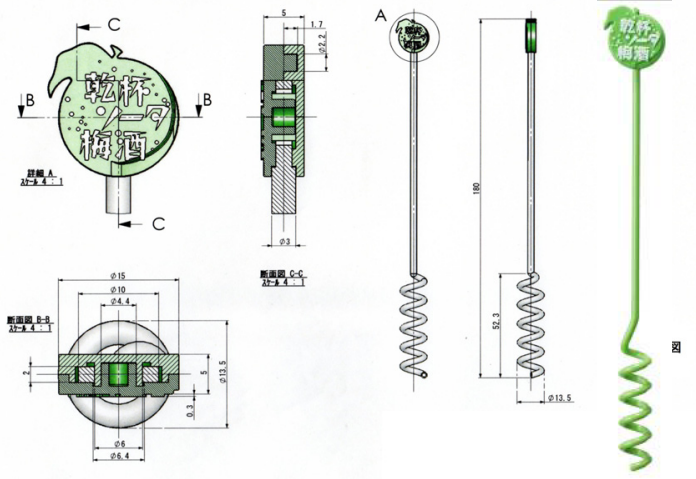

Figure 6. The blueprint and finished goods of the muddler

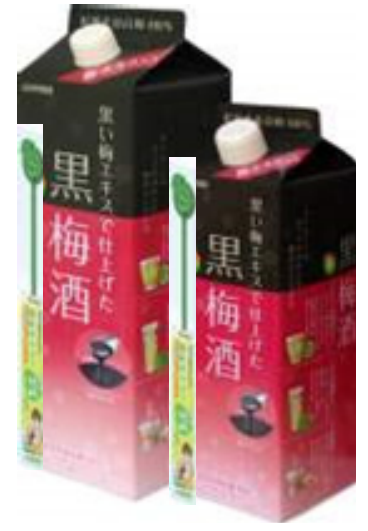

Figure 7. Packaging of goods is written. Here, the busy condition of the muddler was also shown.

The "Kanpai-muddler" was the schedule of 20,000 piece creation in the first plan. However, since it was reputable, additional creation of the " Kanpai-muddler " was performed, and 80,000 totals were created.

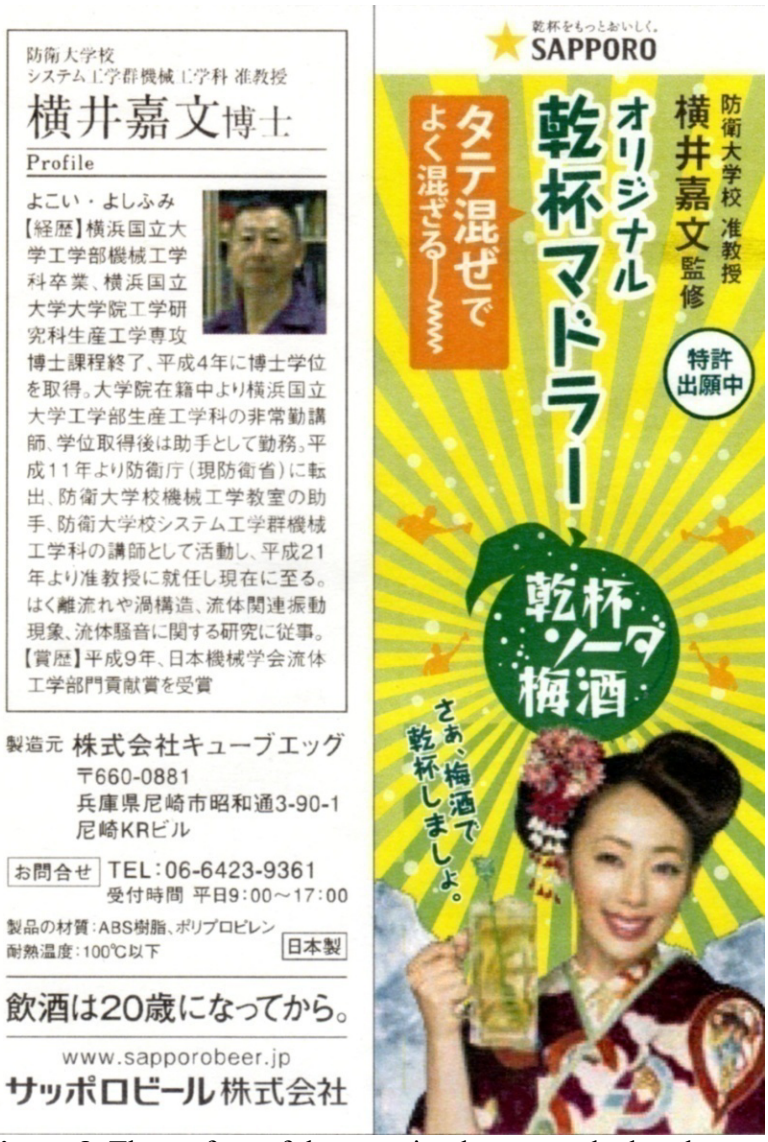

Figure 8. The surface of the covering letter attached to the muddler

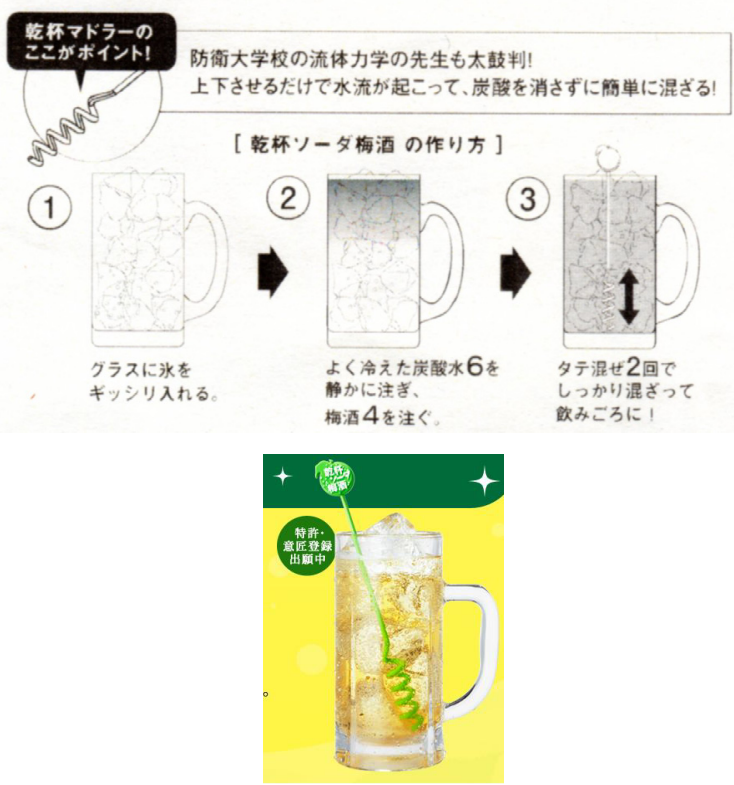

Figure 9. The back of the covering letter attached to the muddler and the busy condition of the muddler 